\title{
Zur Theorie der Trägheitsmomente und der Drehung um einen Punkt.
}

(Von Herrn A. Clebsch zu Carlsruhe.)

Die Theorie der Trägheitsmomente läfst sich in eine Beziehung zu der Theorie der confocalen Oberflächen setzen, welche einige bemerkenswerthe Folgerungen nit sich führt. Bezeichnen wir durch $\boldsymbol{M}$ die Masse des Körpers, durch $\boldsymbol{M} \boldsymbol{a}^{2}, \boldsymbol{M} \boldsymbol{b}^{2}, \boldsymbol{M} \boldsymbol{c}^{2}$ die Trägheitsmomente für die drei Hauptaxen, welche man durch den Schwerpunkt legen kann, so dafs $a, b, c$ für diese Hauptaxen diejenigen Längen bezeichnen, denen man bisweilen den Namen der Trägheitsradien beigelegt hat. Sind ferner $\alpha, \beta, \gamma$ die Winkel welche eine durch den Schwerpunkt gelegte Drehungsaxe mit den Hauptaxen bildet, so ist das Trägheitsmoment für diese Axe bekanntlich

$$
\text { (1.) } \quad \mathrm{M}=\boldsymbol{M}\left(\boldsymbol{a}^{2} \cos ^{2} \alpha+\boldsymbol{b}^{2} \cos ^{2} \beta+\boldsymbol{c}^{2} \cos ^{2} \gamma\right) \text {. }
$$

Ist ferner $\varrho$ der Trägheitsradius für diese Axe und sind $u, v, w$ die Ebenencoordinaten derjenigen Ebene, welche die Drehungsaxe zur Normale hat und vom Schwerpunkte um $\rho$ entfernt ist, so hat man die Gleichungen

$$
\text { (2.) } \cos \alpha=-\varrho u, \quad \cos \beta=-\rho v, \quad \cos \gamma=-\varrho w,
$$

und die Gleichung (1.) geht über in:

$$
\text { (3.) } \quad 1=a^{2} u^{2}+b^{2} v^{2}+c^{2} w^{2} \text {. }
$$

Dies ist die Gleichung eines Ellipsoides, dessen Hauptaxen $a, b, c$ sind, und welches in Punktcoordinaten die Gleichung hat:

$$
\text { (4.) } \quad 1=\frac{x^{2}}{a^{2}}+\frac{y^{2}}{b^{2}}+\frac{z^{2}}{c^{2}} \text {. }
$$

Dies Ellipsoid, welches dem Folgenden zu Grunde gelegt werden soll, will ich das zweite Centralellipsoid nennen, im Gegensatz zu dem sonst betrachteten, dessen Radien den Quadratwurzeln der reciproken Trägheitsmomente proportional sind. Man hat also zunächst den Satz:

\section{Satz 1.}

Das Loth, welches vom Schuerpunkle auf eine Tangentenebene dieses Ellipsoides gefällt wird, ist gleich dem Trägheitsradius für die Drehungsaxe, welche das Loth selbst darstellt. 
Ich will jetzt die Hauptaxen aufsuchen, welche man durch irgend einen anderen Punkt des Körpers führen kann. Sind die Coordinaten dieses Punktes $x, y$, so wird der Ausdruck für das Trägheitsmoment einer Axe, welche durch ihn gelegt ist, erhalten, indem man zu (1.) den Ausdruck $\boldsymbol{M d}^{2}$ hinzufügt, wo $d$ den Abstand der neuen Drehungsaxe vom Schwerpunkte ausdrückt. Daher nimmt der neue Trägheitsradius den Werth an:

(5.) $\rho^{2}=a^{2} \cos ^{2} \alpha+b^{2} \cos ^{2} \beta+c^{2} \cos ^{2} \gamma+\left(x^{2}+y^{2}+z^{2}\right)-(x \cos \alpha+y \cos \beta+z \cos \gamma)^{2}$, oder, wenn man $x^{2}+y^{2}+z^{2}=r^{2}$ setzt und wieder Ebenencoordinaten einführt, für welche der Punkt $(x, y, z)$ der Anfangspunkt der Coordinaten ist,

(6.) $1=\left(a^{2}+r^{2}\right) u^{2}+\left(b^{2}+r^{2}\right) v^{2}+\left(c^{2}+r^{2}\right) w^{2}-(u x+v y+w z)^{2}$.

Dies ist wiederum die Gleichung eines Ellipsoides, welches den Punkt $(x, y, z)$ zum Mittelpunkt und die Trägheitsradien der durch ihn gelegten Axen zu Normalen der Tangentenebenen hat. Um die Hauptaxen dieses Ellipsoides oder die Richtungen der Drehungsaxen mit gröfsten und kleinsten Trägheitsmomenten zu finden, ist nur der Ausdruck

$$
u^{2}+v^{2}+w^{2}=\frac{1}{\rho^{2}}
$$

zu einem Minimum zu machen. Dies giebt für die Richtung und Gröfse des fraglichen Radius aus (5.):

$$
\begin{aligned}
& \left(a^{2}+r^{2}-\rho^{2}\right) \cos \alpha=x(x \cos \alpha+y \cos \beta+z \cos \gamma), \\
& \left(b^{2}+r^{2}-\rho^{2}\right) \cos \beta=y(x \cos \alpha+y \cos \beta+z \cos \gamma), \\
& \left(c^{2}+r^{2}-\rho^{2}\right) \cos \gamma=z(x \cos \alpha+y \cos \beta+z \cos \gamma),
\end{aligned}
$$

wodurch sich die cubische Gleichung für $\varrho^{2}$ ergiebt:

während

$$
1=\frac{x^{2}}{a^{2}+r^{2}-\varrho^{2}}+\frac{y^{2}}{b^{2}+r^{2}-\varrho^{2}}+\frac{z^{2}}{c^{2}+r^{2}-\varrho^{2}},
$$

(8.) $\quad \cos \alpha: \cos \beta: \cos \gamma=\frac{x}{a^{2}+r^{2}-\varrho^{2}}: \frac{y}{b^{2}+r^{2}-\varrho^{2}}: \frac{z}{c^{2}+r^{2}-\varrho^{2}}$.

Daher hat man den folgenden Satz:

\section{Satz 2.}

Die Hauptaxen des Ellipsoides (6.), d. h. die Maximumsurerthe der Trägheitsradien für den Punkt $(x, y, z)$ fallen in die Richtung der Normalen der drei zu dem Centralellipsoid (4.) confocalen Oberflächen, welche sich durch diesen Punkt legen lassen. Die Grö/sen dieser Haupt- 
axen sind $\sqrt{r^{2}+\lambda^{2}}, \sqrt{r^{2}+\mu^{2}}, \sqrt{r^{2}+v^{2}}$, wenn $\lambda, \mu, \nu$ die Paraneter der drei confocalen Oberflächen sind, und $r$ die Entfernung des gegehenen Punktes vorn Schwerpunkt bedeutet.

Aus der Form der Gleichung (7.) folgt ferner, in Bezug auf die Gröfse der Hauptträgheitsradien, indem $a>b>c$ vorausgesetzt wird:

\section{Satz 3 .}

Von den Hauptträgheitsradien für den Punkt $(x, y, z)$ ist. immer einer kleiner als $\sqrt{c^{2}+r^{2}}$, ein zweiter liegt zwischen $\sqrt{c^{2}+r^{2}}$ und $\sqrt{b^{2}+r^{2}}$, der dritte wwischen $\sqrt{b^{2}+r^{2}}$ und $\sqrt{a^{2}+r^{2}}$.

Man kann aber das Ellipsoid (6.) seinerseits als Basis eines confocalen Systems ansehen und die drei diesem confocalen Oberflächen aufsuchen, welche durch den Schwerpunkt, den Mittelpunkt des ersten Systems, gelegt werden können. Nach einem bekannten Reciprocitätsgesetze, welches für solche Systeme gültig ist, kann man dann den Satz aufstellen:

Satz 4.

Kennt man das Ellipsoid (6.) für irgend einen Punkt des Körpers und führt drei ihm confocale Oberflächen durch den Schujerpunkt, so sind die Normalen dieser drei Oberflüchen die Richtungen der Hauptaxen des Schwerpunktes und wenn $l, m, n$ die Parameter dieser Oberflächen bezeichnen, so sind $\sqrt{l^{2}-r^{2}}, \sqrt{m^{2}-r^{2}}, \sqrt{n^{2}-r^{2}}$ die Werthe der Hanpttrügheitsradien für den Schwerpunkt.

Diese Construction gilt auch noch, wenn man für den Schwerpunkt einen beliebigen Punkt annimmt, welcher auf der Verbindungslinie desselben mit dem gegebenen Punkte liegt. Nur ist dann an die Stelle des Ellipsoides (6.) ein anderes zu setzen, welches ihm ähnlich ist.

Die Gleichung (7.) ist noch in einer anderen Weise bemerkenswerth. Sucht man nämlich diejenigen Punkte des Körpers auf, welche einen Hauptträgheitsradius gemeinsam haben, so hat man in der Gleichung (7.) nur $\rho$ als constant zu betrachten. Diese Gleichung stellt dann eine Oberfläche von dernselben Character dar uie die in der Optik betrachtete Oberfläche, deren Radien die Fortpflanzungsgeschwindigkeiten der Strahlen in krystullinischen Medien angeben; und während man dem Parameler verschiedene Werthe beilegt, erhält man ein System von gewissermafsen confocalen Oberflächen dieser Art, welche sich in drei vollkommen gesonderte Klassen 
scheiden, jenachdem $\rho^{2}$ zwischen $c^{2}$ und $b^{2}$ oder zwischen $b^{2}$ und $a^{2}$ liegt, oder endlich gröfser als $a^{2}$ ist. Die in der Optik betrachtete Oberfläche gehört zu der letzten Klasse.

Sucht man nun solche Punkte des Körpers auf, für welche zwei Hauptträgheitsradien einander gleich werden, so müssen für diese Punkte die Gleichungen (8.), welche die Richtungen der Hauptaxen angeben, unbestimmt werden, d. h. es mufs einer der Fälle eintreten:

$$
\begin{aligned}
& x=0^{k} \text { und } a^{2}+r^{2}-\rho^{2}=0 \text { oder } \\
& y=0 \text { und } b^{2}+r^{2}-\rho^{2}=0 \text { oder } \\
& z=0 \text { und } c^{2}+r^{2}-\rho^{2}=0 \text {. }
\end{aligned}
$$

Die Curve, auf welcher der Punkt $(x, y, z)$ liegen mufs, hat dann also eins der folgenden drei Systeme zu Gleichungen:

$$
\begin{aligned}
& x=0 \text { und } 1=\frac{y^{2}}{b^{2}-a^{2}}+\frac{z^{2}}{c^{2}-a^{2}} \quad \text { oder } \\
& y=0 \text { und } 1=\frac{z^{2}}{c^{2}-b^{2}}+\frac{x^{2}}{a^{2}-b^{2}} \quad \text { oder } \\
& z=0 \text { und } 1=\frac{x^{2}}{a^{2}-c^{2}}+\frac{y^{2}}{b^{2}-c^{2}} .
\end{aligned}
$$

Diese Curven, von denen die erste imaginär, die zweite eine Hyperbel, die dritte eine Ellipse ist, sind keine andern, als die Focallinien der Oberfläche (4.). Man hat also den Satz:

Satz 5.

Diejenigen Punkte, für welche zwei Hauptträgheitsmomente gleich werden, bilden die Focallinien des Centralellipsoides (4.). Und zwar sind, wie aus (8.) folgt, die Richtungen der beiden gleichen Hauptaxen. die Tangente der Focalcurve und eine Axe des Ellipsoides (4.), während die ungleiche Axe die Normale der Focallinie im Hauptschnitte wird.

Man bemerkt leicht, da/s dieselben Punkte zugleich die Reihe von Doppelpunkten constituiren, welche das Oberflächensystem (7.) aufiveist. Die Doppelpunkte der optischen Oberflächen bilden die Focalhyperbel; die Focalellipse gehört denjenigen Oberflächen an, für welche $\varrho^{2}$ zwischen $b^{2}$ und $a^{2}$ liegt. Für die Oberflächen endlich, bei denen $\varrho^{2}$ zwischen $c^{2}$ und $b^{2}$ liegt, werden sämmtliche Doppelpunkte imaginär.

Für die Punkte der Focalhyperbel sind die gröfseren Trägheitsmomente einander gleich, für die Punkte der Ellipse die kleineren. Man sieht, dafs 
alle drei Hauptträgheitsmomente nur dann einander gleich werden können, wenn diese beiden Curven einen Punkt, d. h. ihren Scheitel, gemeinsam haben, oder wenn $b=c$ ist. Alsdann gehen zugleich die Focallinien in ein Punktenpaar über, welches durch $x=\sqrt{a^{2}-b^{2}}$ gegeben ist. Dies ist ein Resultat, welches sehr bekannt ist, aber auf ganz anderem Wege gewonnen zu werden pflegt.

Das Ellipsoid (3.) oder (4.) hängt mit dem Poinsotschen Centralellipsoide sehr genau zusammen, so dafs vermöge des geometrischen Princips der Reciprocität die für das eine gewonnenen Re ultate sich auf das andere leicht übertragen lassen. Dies führt zu einigen neusn Sätzen über die Drehung eines Körpers um einen festen Punkt, wenn keine Kräfte auf den Körper wirken. Einige der hauptsächlichsten dieser Sätze, welche den von Poinsot aufgestellten ganz analog werden, sind folgende:

1. Zieht man in dem Ellipsoide (4.) denjenigen Radius vector, der die Axe des mittleren Paares der Bewegungsgrö/sen darstellt, so ist die ihr conjugirte Ebene oder die in seinem Endpunkte gelegte Tangentenebene die Ebene der Drehung des Körpers, ihre Normale die augenblickliche Drehungsaxe.

2. Die augenblickliche Winkelgeschwindigkeit ist dem auf diese Tangentenebene gefültten Lothe umgekehrt proportional.

3. Die Länge des betrachteton Radius vector und seine Lage im Raume bleibt während der ganzen Bewegung constant. Es giebt also einen gewissen Kegel, welcher durch den Schnitt des Ellipsoides (4.) mit einer Kugel hindurchgeht, dessen Seiten successive die Stelle dieses Radius vector einnehmen. (Dieser Satz findet sich schon bei Poinsot).

4. Die auf einander folgenden Drehungsebenen des Körpers sind die Tangentenebenen einer abwickelbaren Oberfläche, welche gleichzeitig zwei Oberfächen zueiter Ordnung mit gemeinschuflichem Mittelpunkte und gleich gerichteten Hauptaxen berührt, von denen eine das Ellipsoid (4.) ist. Diese Oberfläche schneidet jede der im Körper festen Coordinatenebenen in eivem Kegelschnitte.

5. Dieselben Drehungsebenen umhüllen im Raume einen transscendenten Kegel mit unendlich vielen Windungen, dessen Spitze in den Endpunkt des unter 1. 3. erwähnten Radius fällt. Dieser Kegel entspricht der Serpoloide Poinsots.

Carlsruhe, den 22. Februar 1859. 